\title{
Electrocardiogramas durante el tercer trimestre de embarazo: descripción de sus características
}

\author{
Electrocardiography During the Third Trimester of Pregnancy: Description of its \\ Characteristics
}

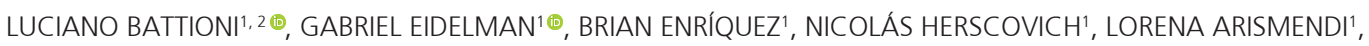
ALEJANDRO DEVIGGIANO'

RESUMEN

Objetivo: Analizar las características electrocardiográficas en embarazadas sin patología cardiovascular. Material y métodos: Estudio de corte transversal, descriptivo, multicéntrico. Se incluyeron pacientes sin patología cardiovascular que cursaban el tercer trimestre de embarazo y que concurrieron a una evaluación cardiológica preparto entre abril y julio de 2020; todas ellas firmaron el consentimiento correspondiente.

Resultados: Se analizaron 80 trazados. La mediana de la frecuencia cardíaca fue 82 lpm (RIC: 70-93 lpm). La mediana del eje QRS fue $54^{\circ}$ (RIC: $39^{\circ}-71^{\circ}$ ). Ondas q e infradesnivel del segmento ST en cara inferior y de V4 a V6 fueron hallazgos relativamente frecuentes. La mediana del QTc fue $422 \mathrm{mseg}$ (RIC: $404 \mathrm{mseg}-445 \mathrm{mseg}$ ). La mediana del tiempo del pico de la onda T a su fin fue $86 \mathrm{mseg}$ (RIC: 74-95 mseg).

Conclusión: Las alteraciones más frecuentes ocurrieron en DIII, DII, aVf y de V4 a V6. Las ondas q y el infraST fueron los cambios principales. Fueron infrecuentes las desviaciones del eje, la taquicardia sinusal o el QTc prolongado.

Palabras clave:Complicaciones cardiovasculares del embarazo - Tercer trimestre del embarazo - Electrocardiografía - Enfermedades cardiovasculares

\begin{abstract}
Objective: The aim of this study was to analyze the electrocardiographic characteristics in pregnant women without cardiovascular disease.

Methods: This was a descriptive, cross-sectional, multicenter study, including patients without cardiovascular disease in their third trimester of pregnancy, who underwent cardiac evaluation before delivery between April and July 2020. All patients signed the corresponding informed consent.

Results: A total of 80 tracings were analyzed. Median heart rate was $82 \mathrm{bpm}(\mathrm{IQR} 70-93 \mathrm{bpm})$ and median QRS axis was $54^{\circ}$ $\left(\mathrm{IQR} 39^{\circ}-71^{\circ}\right)$. Q waves and ST segment depression were relatively frequent in inferior leads and from V4 to V6. Median QTc was $422 \mathrm{msec}$ (IQR 404-445 msec) and median time from T wave peak to T wave end was $86 \mathrm{msec}$ (IQR 74-95 msec).

Conclusion: The most common changes occurred in T wave peak to T wave end and from V4 to V6. Main changes included $\mathrm{q}$ waves and ST-segment depression. Axis deviations, sinus tachycardia or prolonged QTc were rare.
\end{abstract}

Key words: Pregnancy Complications, Cardiovascular - Pregnancy Trimester, Third - Electrocardiography - Cardiovascular disease

\section{INTRODUCCIÓN}

La enfermedad cardiovascular (CV) tiene manifestaciones únicas en la mujer. Este hecho ha tomado relevancia institucional y pública en Argentina y el mundo. Como consecuencia, se han implementado distintas iniciativas por parte de diferentes sociedades científicas como el Área Corazón y Mujer de la SAC.

El embarazo impone cambios fisiológicos en el sistema CV, que tienen como objetivo la adecuada perfusión de los órganos maternos y el feto. Este representa un período único de vulnerabilidad cardiovascular, con necesidades no satisfechas de diagnóstico y tratamiento.
A pesar de haber sido ampliamente descritos, algunos de estos cambios pueden confundirse con patología CV.

En el electrocardiograma (ECG) de superficie, se han reportado alteraciones del ST-T y de la repolarización,también aparición de ondas Q. Estos cambios se profundizan durante el tercer trimestre (3. er TM). Estas características están documentadas en publicaciones anteriores, pero se limitan al análisis de series de menos de 100 pacientes en poblaciones asiáticas y con una edad promedio de 25 años. (4-9)

El objetivo del presente estudio fue evaluar en la población local las características clínicas y del ECG de embarazadas sin enfermedad cardiovascular previa

Rev Argent CARDIol 2021;89:135-139. http://dx.doi.org/10.7775/rac.es.v89.i2.20011

Recibido: 26/11/2020 - Aceptado: 18/02/2021

Dirección para separatas: Luciano Battioni - Calle 3 n3070, CP 6600, Mercedes - Prov. de Bs. As. - E-mail: lucianobattioni@gmail.com

Fuentes y apoyo: No se recibieron donaciones ni apoyos financieros

\footnotetext{
${ }^{1}$ Diagnóstico Maipú, Servicio de Cardiología
}

${ }^{2}$ Sección Cardiología Medbinars.com 
que cursan el 3. ${ }^{\text {er }}$ TM. Además se consideraron las características diferenciales según la edad dicotomizada en 40 años.

\section{MATERIAL Y MÉTODOS}

Se realizó un estudio de corte transversal, descriptivo, multicéntrico. Se incluyeron pacientes sin patología CV previa

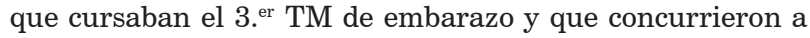
la evaluación cardiológica preparto en los consultorios de Diagnóstico Maipú, sedes Pilar y Cabildo, entre abril y julio de 2020.Todas ellas habían firmado el consentimiento para el uso de sus datos para investigación.

La evaluación clínica fue realizada por un médico especialista en cardiología, a través de un formulario estandarizado para recabar antecedentes y factores de riesgo cardiovasculares y de un examen físico.

La toma de los ECG se realizó según técnica estándar de 12 derivaciones, con una velocidad de barrido de $25 \mathrm{~mm} / \mathrm{seg}$ y una amplitud de $0,1 \mathrm{~mm} / \mathrm{mV}$ en el formato $6 \times 2$ con una tira de ritmo DII largo debajo en un dispositivo de adquisición digital (ECGview, ECCOSUR, Córdoba, Argentina). El ECG fue analizado offline por el primer y el segundo autor utilizando el cáliper electrónico. Las diferencias en mediciones se acordaron sobre la base del consenso.

Las mediciones se realizaron ajustando la velocidad y la escala según requerimiento. El intervalo QT se midió tomando como inicio la primera deflexión del QRS y el fin de la onda T. El fin de la onda T se definió usando la técnica de intersección de la línea isoeléctrica con la tangente de la máxima pendiente de la onda $\mathrm{T}$.

\section{Análisis estadístico}

Las variables categóricas se expresaron en frecuencias absolutas y relativas. Las numéricas en promedio y desvío estándar o mediana e intervalo intercuartílico, según su distribución. Las diferencias entre las variables numéricas se evaluaron mediante U de Mann Whitney y las categóricas mediante el test de Chi cuadrado o exacto de Fisher, según correspondiera.

\section{Consideraciones éticas}

El estudio fue aprobado por el Comité de Ética e Investigación Institucional. Se estableció que no se requeriría consentimiento adicional al que firmaron las pacientes al concurrir al establecimiento, dado que es un estudio retrospectivo, sin intervención, y que todas habían firmado un consentimiento donde se les consultaba expresamente si sus datos podían ser usados para investigación clínica.

\section{RESULTADOS}

Se incluyeron 80 pacientes que se hallaban cursando una mediana de 35 semanas de edad gestacional (SEG) (RIC: $33-36$ semanas), 10 (12,5\%) de las cuales eran mayores de 40 años. Las características clínicas se resumen en la Tabla 1.

La mediana de frecuencia cardíaca (FC) fue 82 lpm (RIC: 70-93 lpm). La mediana del eje QRS fue $54^{\circ}$ (RIC: $39^{\circ}-71^{\circ}$ ) (Tabla 2). Dos pacientes presentaron desviaciones del eje mayores de $90^{\circ}$ y 2 menores de $0^{\circ}$. La morfología SDI QDIII T-DIII se observó en 5 gestantes $(6,3 \%)$. Se observaron en cara inferior ondas q (DII 37,5\%; DIII 46,3\%;aVF43,8\%) e infradesnivel del segmento ST (DII 23,8\%; DIII 15\%; a VF22,5\%). En la cara lateral baja la prevalencia de ondas q fue de $10 \%$

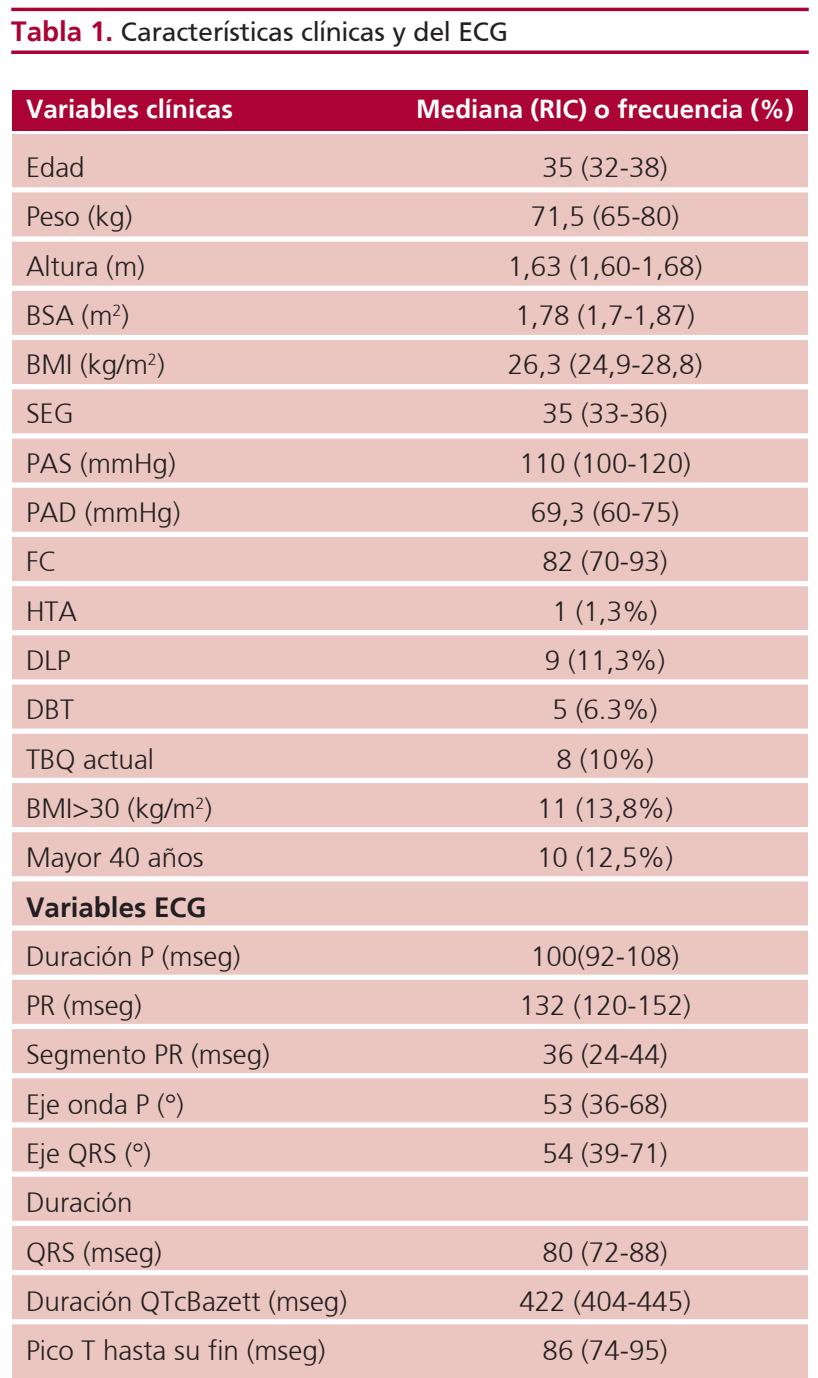

SC: Superficie Corporal; IMC: Índice de Masa Corporal; SEG: semanas de edad gestacional; PAS: presión arterial sistólica; PAD: presión arterial diastólica; FC: frecuencia cardíaca; HTA: hipertensión; DLP: dislipidemia; DBT: diabetes; TBQ: tabaquista

en V4, 23,8\% en V5 y 25\% en V6; y la de infradesnivel del segmento ST de $13,8 \%, 12,5 \%$ y $13,8 \%$ respectivamente. En la cara lateral baja nunca se observaron ondas q y alteraciones del ST de manera simultánea. Tampoco hubo alteraciones en V4-V5 sin que V6 tuviera idénticas alteraciones.

Se observaron $\mathrm{T}$ negativas predominantemente en V1 y DIII, $80 \%$ y 16,3\%, respectivamente. De manera menos frecuente se observaron estos cambios en V2 $(7,5 \%)$ y V3(1,3\%). Las alteraciones con frecuencia mayor de $10 \%$ se observan en la Figura 1.

La mediana del QTc por fórmula de Bazett fue 422 mseg (RIC: 404 -445 mseg). La mediana del tiempo del pico de la onda T a su fin fue $86 \mathrm{mseg}$ (RIC: $74-95 \mathrm{mseg}$ ).

Se incluyeron 10 pacientes mayores de 40 años. La edad mediana fue 42 años (RIC: 40-44 años). A excepción del antecedente de diabetes (DBT) (3 de 10 ptes. 
Tabla 2. Características clínicas y del ECG de las pacientes de acuerdo a su edad

Fig. 1. Cambios por derivación observados con una frecuencia mayor del $10 \%$

\begin{tabular}{|c|c|c|c|}
\hline & $\begin{array}{l}\text { Menores de } 40 \text { años } \\
(N=70)\end{array}$ & $\begin{array}{l}\text { Mayores de } 40 \text { años } \\
(\mathrm{N}=10)\end{array}$ & $p=$ \\
\hline Edad & $34(31-37)$ & $42(40-44)$ & 0,001 \\
\hline SEG & $35(33-36)$ & $33(32-35)$ & 0,237 \\
\hline HTA & $1(1 \%)$ & $0(0 \%)$ & 1 \\
\hline DLP & $8(11 \%)$ & $1(10 \%)$ & 1 \\
\hline DBT & $2(3 \%)$ & $3(30 \%)$ & 0,013 \\
\hline TBQ & $7(10 \%)$ & $1(10 \%)$ & 0,5 \\
\hline Peso (kg) & $71(64-80)$ & $75(66-83)$ & 0,528 \\
\hline Altura $(\mathrm{cm})$ & $163(160-168)$ & $162(158-167)$ & 0,414 \\
\hline $\mathrm{IMC}\left(\mathrm{kg} / \mathrm{m}^{2}\right)$ & $26(25-28)$ & $28(25-32)$ & 0,335 \\
\hline $\mathrm{SC}\left(\mathrm{m}^{2}\right)$ & $1,78(1,7-1,9)$ & $1,82(1,72-1,86)$ & 0,801 \\
\hline PAS (mmHg) & $110(101-120)$ & $110(100-120)$ & 0,789 \\
\hline PAD (mmHg) & $70(60-75)$ & $70(60-80)$ & 0,885 \\
\hline FC & $84(71-94)$ & $79(67-82)$ & 0,128 \\
\hline Duración P (mseg) & $100(92-108)$ & $94(91-116)$ & 0,866 \\
\hline Duración PR (mseg) & $132(120-152)$ & $136(115-157)$ & 0,252 \\
\hline Segmento PR & $36(24-46)$ & $40(20-44)$ & 0,769 \\
\hline $\operatorname{Eje} P\left({ }^{\circ}\right)$ & $53(40-68)$ & $50(28-70)$ & 0,535 \\
\hline Eje QRS (msg) & $59(39-73)$ & $42(17-71)$ & 0,310 \\
\hline Duración QRS $\left(^{\circ}\right)$ & $80(71-90)$ & $76(71-84)$ & 0,668 \\
\hline Duración QTc Bazett (mseg) & $422(404-441)$ & $436(393-448)$ & 0,996 \\
\hline $\begin{array}{l}\text { Duración onda T desde su pico } \\
\text { hasta el fin (mseg) }\end{array}$ & $86(73-96)$ & $86(79-95)$ & 0,707 \\
\hline
\end{tabular}

Las variables continuas se expresan en mediana y RIC;las categóricas en frecuencias absolutas y relativas.

SG: semanas de edad gestacional; HTA: hipertensión; DLP: dislipidemia; DBT: diabetes; TBQ: tabaquista; IMC: Índice de Masa Corporal; SC: Superficie Corporal; PAS: presión arterial sistólica; PAD: presión arterial diastólica; FC: frecuencia cardíaca

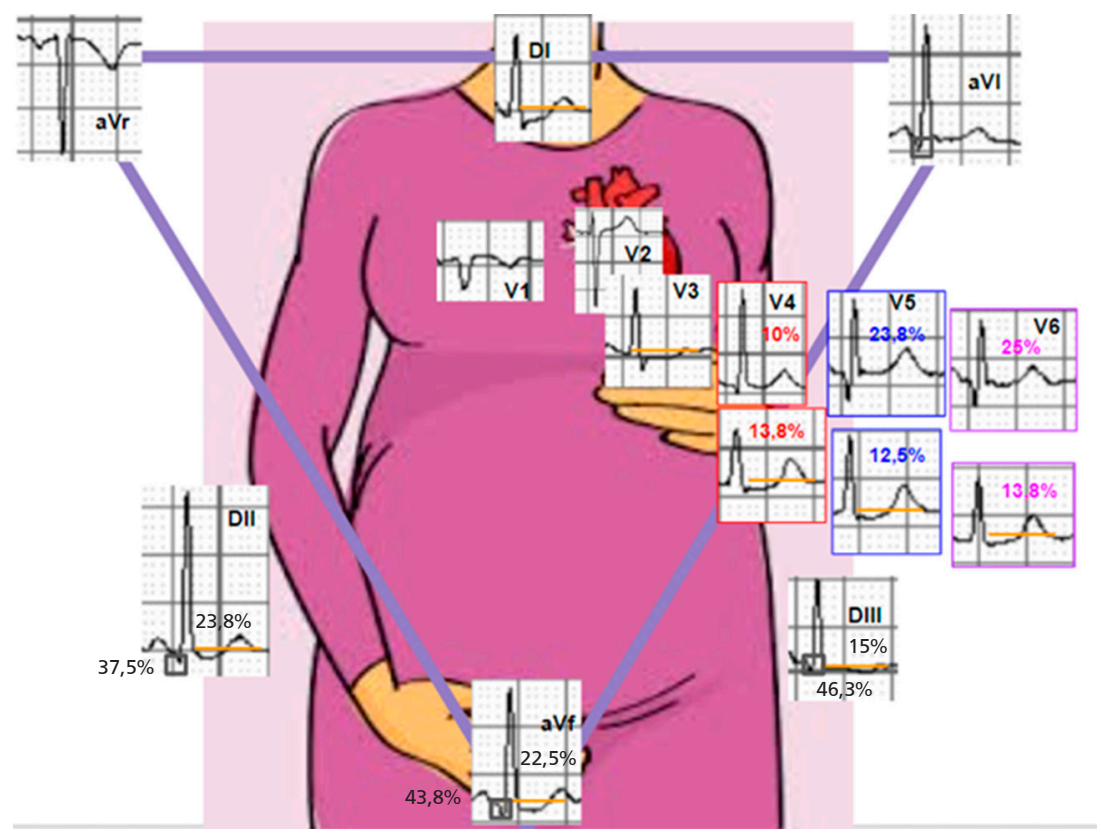


entre las mayores de 40 años vs. 2 ptes. de 70 ptes. entre las menores de 40 años; $p=0,013$ ), la distribución de comorbilidades y variables electrocardiográficas no fueron diferentes significativamente. No hubo diferencias en la presencia de ondas q, infradesnivel del ST o alteraciones de las ondas $\mathrm{T}$.

\section{DISCUSIÓN}

En nuestro estudio observamos la ausencia de los cambios tradicionalmente asociados al embarazo: taquicardia sinusal, eje QRS horizontalizado o desviado a la izquierda. Los cambios encontrados más frecuentemente incluyen ondas q en cara inferior y lateral baja, así como también infradesnivel del segmento ST de hasta $1 \mathrm{~mm}$ en dichas derivaciones. Las pacientes embarazadas mayores de 40 años presentaron mayor prevalencia de DBT, pero no hubo diferencias en los parámetros del ECG cuando se las comparó con gestantes de menor edad.

La frecuencia cardíaca mediana en nuestro estudio fue $82 \mathrm{lpm}$. Solamente 9 sobrepasaron los $100 \mathrm{lpm}$. Esto es diferente de lo publicado por Adamsonet al. (4) y Sumalatha et al. (10), quienes reportaron que la taquicardia sinusal fue el hallazgo más frecuente y la FC promedio fue de $100 \mathrm{lpm}$, respectivamente. En concordancia con nuestros hallazgos, otros autores han publicado frecuencias cardíacas promedio entre $80 \mathrm{y}$ $85 \mathrm{lpm}$. (6,9) El aumento en la frecuencia cardíaca es uno de los mecanismos utilizados para incrementar el gasto cardíaco. Este incremento se debe a la disminución del tono parasimpático (11) y al aumento de la automaticidad de la corriente hiperpolarizante activadora no selectiva If. Sin embargo, todavía no hay explicaciones de por qué ocurren estos cambios, ya que los estudios realizados para dilucidar el efecto de las hormonas gestacionales en el músculo cardíaco han arrojado resultados contradictorios. (7)

$\mathrm{El}$ eje del QRS no fue significativamente diferente de lo observado por Sunitha et al. (5),quienes hallaron que el promedio del eje se encontraba en $46^{\circ}$, o de la media del eje en el $3 .^{\text {er }} \mathrm{TM}$ de $40^{\circ}$ descripta por Carruth et al. (8). Habitualmente se considera que el corazón de la paciente grávida se encuentra horizontalizado por efecto del útero; sin embargo, parecería ser que, a pesar de esto, no se alejaría de los valores esperados en una paciente no embarazada.

La presencia de una onda q en DIII es aceptada como normal en la mayoría de las personas, en ausencia de cambios acompañantes en otras derivaciones contiguas. En nuestro estudio observamos que entre el 30\% y el $40 \%$ de las pacientes tenían onda q en cara inferior, a veces acompañada de cambios en la onda T. Esto también fue observado por Chaudhary et al. (9),quienes informaron que hasta el 30\% de las pacientes presentaban ondas q en DIII, DII y aVF. Similares hallazgos reportaron Sunitha et al. (5) La presencia de ondas q en las derivaciones precordiales, desde V3 hasta V6, ha sido descrita por ambos autores, pero con frecuencias dispares, entre el $10 \%$ y el $60 \%$. En nuestro estudio, la frecuencia de ondas q fue creciente de V4 a V6 y no superó el $25 \%$ en esta última derivación. También, observamos que nunca hubo ondas q en las precordiales si no había una onda q en V6, así como tampoco se observaron ondas q de manera simultánea con alteraciones del ST; esta observación podría ser útil para distinguir ondas q asociadas a patología estructural; sin embargo, se requiere más investigación. La génesis de las ondas q sigue siendo materia de discusión; en nuestro estudio, no podemos atribuirlas a la desviación del eje a la izquierda.

Otro hallazgo de este estudio es la ocurrencia de infradesnivel del segmento ST en todas las derivaciones, excepto V2. La mayor frecuencia se observó en DII, aVF y DI; también fue frecuente desde V3 hasta V6. Cuando se utilizó una definición más dura de infradesnivel del segmento del ST ( $-1 \mathrm{~mm})$, su frecuencia cambió a un $10 \%$ en DII y aVF y hasta un $5 \%$ en las precordiales izquierdas. Estos hallazgos se encuentran en contraposición con estudios previos, donde la presencia de infradesnivel del segmento ST fue observada en un solo paciente. (9) Por oposición, la elevación del segmento ST de más de 1mm solo se observó en V1 y V2 de manera infrecuente.

Detectamos la presencia de ondas T invertidas más frecuentemente en las precordiales derechas de V1 a V3, con disminución de su frecuencia a medidas que se alejaban de V1. En los miembros, la presencia de T invertidas en DIII fue la más observada. Esto coincide con lo reportado en estudios previos. (4)

La mediana del tiempo del pico de la onda $\mathrm{T}$ hasta su fin, subrogante de heterogeneidad de repolarización miocárdica, fue de $86 \mathrm{mseg}$, dato coincidente con el aportado en el estudio de Tanindi et al. (6), en que a su vez fue sustancialmente diferente de los controles. A pesar de esto, el QT corregido por la fórmula de Bazett se encontró dentro del intervalo de normalidad en el 72\% de las pacientes. El 18\% de los casos con QT corregido prolongado tuvo una media de $454 \mathrm{mseg}$, en ningún caso sobrepasó los 480 mseg.

Las razones de las alteraciones del QT en el embarazo permanecen discutidas;a partir de estudios basados en modelos animales y humanos, se cree que los estrógenos podrían afectar la repolarización cardíaca. (12) Datos del cambio del QTc durante el ciclo menstrual muestran resultados contradictorios. (13-15)Se requiere un mayor número de estudios para comprender los mecanismos moleculares de estos cambios en la repolarización ventricular.

En nuestro estudio, no observamos diferencias entre los ECG de las embarazadas menores de 40 años y las que superaban esa edad, pero hallamos una pequeña diferencia, aunque significativa estadísticamente, en la prevalencia de DBT. La edad materna avanzada se asocia de manera independiente a eventos adversos maternos y fetales. (16) El 12,5\% de nuestra muestra estuvo constituido por mujeres mayores de 40 años que estaban cursando un embarazo.Si bien en números 
absolutos es una muestra pequeña, esta proporción guarda relación con lo publicado por Claramonte Nieto et al. (17), quienes,además, hallaron una asociación entre DBT y edad materna, en consonancia con nuestros hallazgos. A esto debe sumarse el hecho de que el aumento en la edad gestacional es una tendencia mundial; en algunos países es el único segmento etario con tasas de gestación en ascenso. (18)

Nuestro estudio es observacional en naturaleza y se encuentra sujeto a todos los sesgos inherentes a su diseño. Asimismo, no contamos con grupo control para poder comparar los ECG con una cohorte no gestante.

\section{CONCLUSIÓN}

Los trazados electrocardiográficos de pacientes embarazadas que se encontraban cursando el tercer trimestre de gestación raramente presentaron taquicardia sinusal; la mayoría presentaron el eje del QRS en el primer cuadrante. Fue frecuente la presencia de ondas q en cara inferior y lateral baja, así como también la presencia de infradesnivel del segmento ST. Se observaron T negativas en precordiales derechas. El Qtc se encontró dentro de límites de normalidad o ligeramente aumentado a expensas de un mayor tiempo del pico de la onda $\mathrm{T}$ a su finalización. No hubo diferencias en los trazados cuando se comparó a las pacientes según su edad.

\section{Declaración de conflicto de intereses}

Los autores declaran que no poseen conflicto de intereses.

(Véase formulario de conflicto de intereses de los autores en la web / Material suplementario).

\section{BIBLIOGRAFÍA}

1. Mehta LS, Warnes CA, Bradley E, Burton T, Economy K, Mehran $\mathrm{R}$, et al. Cardiovascular Considerations in Caring for Pregnant Patients: A Scientific Statement From the American Heart Association. Circulation. 2020;141(23):e884-e903. https://doi.org/10.1161/ CIR.0000000000000772

2. Regitz-Zagrosek V, Roos-Hesselink JW, Bauersachs J, BlomströmLundqvist C, Cífková R, De Bonis M, et al. 2018 ESC Guidelines for the Management of Cardiovascular Diseases during Pregnancy. Eur Heart J 2018;39:3165-241. https://doi.org/10.1093/eurheartj/ehy340 3. Fu Q. Hemodynamic and electrocardiographic aspects of uncomplicated singleton pregnancy.AdvExp Med Biol2018;1065:413-31.. https://doi.org/10.1007/978-3-319-77932-4_26

4. Sumalatha B, Jyotsna M, Indrani G. Electrocardiographic Changes during Normal Pregnancy. Indian J Cardiovasc Dis Women WINCARS 2017;02:035-8. https://doi.org/10.1055/s-0037-1606853

5. Sunitha M, Chandrasekharappa S, Brid SV. Electrocardiographic QRS Axis, Q Wave and T-Wave Changes in 2nd and 3rd Trimester of Normal Pregnancy. J ClinDiagn Res 2014;8:BC17-21 https://doi. org/10.7860/jedr/2014/10037.4911

6. Tanindi A, Akgun N, Pabuccu EG, et al. Electrocardiographic PWave Duration, QT Interval, T Peak to End Interval and Tp-e/QT Ratio in Pregnancy with Respect to Trimesters. Ann Noninvasive Electrocardiol2016;21:169-74. https://doi.org/10.1111/anec.12285

7. Bett GC. Hormones and sex differences: Changes in cardiac electrophysiology with pregnancy. ClinSci2016;130:747-59. https://doi. org/10.1042/CS20150710

8. Carruth JE, Mirvis SB, Brogan DR, Wenger NK. The electrocardiogram in normal pregnancy. Am Heart J1981;102:1074-5. https:// doi.org/10.1016/0002-8703(81)90497-X

9. Chaudhary S, Saha CG, Sarkar D. Electrocardiographic Changes During Pregnancy at Third Trimester: A Comparative Study. Med J Shree Birendra Hospital2016;14:41-6. https://doi.org/10.3126/mjsbh. v14i2.13367

10. Adamson DL, Nelson-Piercy C. Managing palpitations and arrhythmias during pregnancy. Heart 2007;93:1630-6 https://doi. org $/ 10.1136 /$ hrt.2006.098822

11. Ekholm EMK, Piha SJ, Erkkola RU, Antila KJ. Autonomic cardiovascular reflexes in pregnancy. A longitudinal study. ClinAuton Res 1994;4:161-5. https://doi.org/10.1007/BF01826181

12. Cheng J. Evidences of the gender-related differences in cardiac repolarization and the underlying mechanisms in different animal species and human. FundamClinPharmacol 2006;20:1-8.https://doi. org/10.1111/j.1472-8206.2005.00384.x

13. Hulot JS, Démolis JL, Rivière R, Strabach S, Christin-Maitre $\mathrm{S}$, Funck-Brentano C. Influence of endogenous oestrogens on QT interval duration. EurHeart J2003;24:1663-7. https://doi.org/10.1016/ S0195-668X(03)00436-6

14. Endres S, Mayuga KA, de Cristofaro A, Taneja T, Goldberger JJ, Kadish AH. Menstrual cycle and ST height. Ann Noninvasive Electrocardiol2004;9:121-6. https://doi.org/10.1111/j.1542-474X.2004.92530.x 15. Nakagawa M, Ooie T, Takahashi N, Taniguchi Y, Anan F, Yonemochi $\mathrm{H}$., et al. Influence of Menstrual Cycle on QT Interval Dynamics.Pacing ClinElectrophysiol2006;29:607-13. https://doi. org/10.1111/j.1540-8159.2006.00407.x

16. Births in England and Wales - Office for National Statistics. Accessed November 9, 2020. https://www.ons.gov.uk/peoplepopulationand community/births deaths and marriages/livebirths/bulletins/birth summary tables englandand wales/latest\#fertility-rates-decreasedfor-all-ages-except-for-women-aged-40-years-and-over

17. Cavazos-Rehg PA, Krauss MJ, Spitznagel EL, BommaritoK, Madden T, Olsen MA, et al. Maternal Age and Risk of Labor and Delivery Complications. Matern Child Health J 2015;19:1202-11. https://doi. org/10.1007/s10995-014-1624-7

18. Claramonte Nieto M, MelerBarrabes E, Garcia Martínez S, Gutiérrez Prat M, Serra Zantop B. Impact of aging on obstetric outcomes: Defining advanced maternal age in Barcelona. BMC Pregnancy Childbirth 2019;19:1-10. https://doi.org/10.1186/s12884-019-2415-3 